\title{
An unusual case of Chronic Lymphocytic Leukemia with trisomy 12 presenting with prolymphocytic transformation and new translocation 8;21.
}

\author{
Mishi Bhushan ${ }^{1}$ and Kirthi Kumar ${ }^{1}$ \\ ${ }^{1}$ Medical City Dallas Hospital
}

November 26, 2020

\author{
Abstract \\ phocytic transformation and new translocation 8;21. \\ First Author: \\ Mishi Bhushan, MD, MPH \\ Pathology observer, Hematopathology \\ Medical City Dallas | Medical City Children's Hospital \\ 7777 Forest Lane, Suite C400 \\ Dallas, TX 75230 \\ Bhushanm0214@gmail.com \\ P: 469-835-9354 \\ Corresponding and Senior Author: \\ Kirthi R. Kumar MD., PhD \\ Medical Director, Hematopathology \\ Forward Pathology Solutions \\ Medical City Dallas | Medical City Children's Hospital \\ 7777 Forest Lane, Suite C400 \\ Dallas, TX 75230 \\ Kirthi.kumar@medicalcityhealth.com \\ P: $972-566-3843$ \\ C: $469-939-5172$
}

Patient with a long history of CLL and known trisomy 12 presented with rapidly rising lymphocytosis, bulky adenopathy, and splenomegaly. Peripheral blood and bone marrow exam showed preponderance of prolymphocytes. Cytogenetic analysis showed an abnormal male karyotype with trisomy 12 and a new $\mathrm{t}(8 ; 21)$ translocation in the same 6 metaphases.

An unusual case of Chronic Lymphocytic Leukemia with trisomy 12 presenting with prolym- 
F: $972-566-3479$

Keywords: CLL, translocation 8;21, AML, RUNX1-RUNX1TX, prolymphocytic leukemia

Key Clinical Message: First report of translocation 8;21 in a patient with CLL. RUNX1-RUNX1T1 fusion gene resulting from the translocation may have played a role in the prolymphocytic transformation.

An 81-year old male with a history of chronic lymphoid leukemia (CLL)/small lymphocytic lymphoma (SLL) for over two decades presented with low back pain, left flank pain, and generalized weakness. Patient's initial diagnostic bone marrow performed in 2005 showed chronic lymphocytic leukemia with trisomy 12 confirmed by routine cytogenetics analysis. Patient had received multiple lines of chemotherapy including ibrutinib for the past three years. Patient's WBC count on this admission was $60.2 \times 10^{9}$ cells/L with hemoglobin $13.2 \mathrm{~g} / \mathrm{dL}$ and platelet count of $95 \times 10^{9}$ cells/L. Imaging studies revealed generalized adenopathy with massive splenomegaly. Peripheral blood smear showed numerous prolymphocytes (Figure 1A). Bone marrow morphology and flow cytometry studies confirmed a prolymphocytic transformation of CLL (Figure 1B). Interestingly, cytogenetics analysis showed an abnormal male karyotype with six out of twenty metaphases showing a translocation between chromosomes 8 and 21 in addition to trisomy 12 (Figure 2A). Two metaphases showed a deletion of the long arm of chromosome 13. Remaining metaphases showed normal karyotype (Figure 2B). Patient was started on chemotherapy with R-EPOCH (rituximab, etoposide, cisplatin, vincristine, cyclophosphamide and prednisone) regimen with clinical response but opted against further therapy after his first cycle of chemotherapy.

CLL/SLL is an indolent lymphoproliferative disorder that can transform into a more aggressive hematolymphoid malignancy in about $5-10 \%$ of patients ${ }^{1,2}$. The histologic transformation can involve an increased number of prolymphocytic cells, referred to as prolymphocytic transformation or into diffuse large cell lymphoma with immunoblastic features, also known as Richter's syndrome (RS) or transformation (RT) ${ }^{3,4}$. RT was first defined in 1928 by Maurice Richter as the transformation of CLL to an aggressive lymphoma such as high-grade non-Hodgkin lymphoma or Hodgkin's lymphoma ${ }^{5-7}$. Transformation into aggressive malignancy clinically presents as new or worsening symptoms such as fevers, night sweats, weight loss, organ failure, and rapidly enlarging lymphadenopathy. It has a highly unfavorable prognosis ${ }^{8-10}$. While transformation to prolymphocytic leukemia has been well documented, there are no known cases to date of transformation to prolymphocytic leukemia with 8;21 translocation. The $\mathrm{t}(8 ; 21)$ (q22;q22) translocation is observed in acute myeloid leukemia (AML) M2 subtype ${ }^{11,12}$. The $\mathrm{t}(8 ; 21)$ translocation results in a fusion oncoprotein between RUNX1 and $\mathrm{ETO}^{13}$. The finding of $\mathrm{t}(8 ; 21)(\mathrm{q} 22 ; \mathrm{q} 22.1) ; \mathrm{RUNX} 1-\mathrm{RUNX} 1 \mathrm{~T} 1$ in the setting of high grade transformation of CLL/SLL is rather unusual with no literature citations of this abnormality.

RUNX1-RUNX1T1 fusion leads to disruption of normal function of the core-binding factor in the regulation of hematopoietic differentiation and maturation. There are associated cooperating mutations in theKIT, FLT3 and possibly JAK2, resulting in activation of oncogenes and signaling pathways in leukemogenesis of AML. The fusion gene also interacts with micro-RNAs in leukemogenesis. RUNX1 is critical for maturation of a wide range of hematopoietic stem cells including myeloid, $\mathrm{B}$ cells and $\mathrm{T}$ cells and has been implicated in pathogenesis of several myeloid and lymphoid malignancies ${ }^{14,15,16}$. We postulate that the new translocation 8; 21 had a role to play in the prolymphocytic transformation of CLL in this patient ${ }^{12}$, but the long interval between the patient's initial karyotyping and at the time of transformation makes this association less definitive.

\section{Author Contributions:}

I, Mishi Bhushan, MD, MPH, am the first author of this publication and Kirthi Raman Kumar, MD, PhD is the advising author of this publication.

Conflict of interest: none

Funding: None

Ethical approval: n/a 
Acknowledgements: Vikas Bhushan, MD for assistance and support as well as access to patient file.

\section{References}

1. Reiniger L, Bödör C, Bognár Á, et al. Richter's and prolymphocytic transformation of chronic lymphocytic leukemia are associated with high mRNA expression of activation-induced cytidine deaminase and aberrant somatic hypermutation. Leukemia . 2006;20(6):1089-1095.

2. Foucar K, Rydell RE. Richter's syndrome in chronic lymphocytic leukemia. Cancer . 1980;46(1):118-134.

3. Rana C, Sharma S, Agarwal M. Prolymphocytic and Richter's Transformation in Peripheral Blood: A Case Report and Review of Literature. J Hematol . 2014;3(3):86-88.

4. Jaffe ES, Harris NL, Stein H, Vardiman JW. WHO Classification of Tumors of Hematopoietic and Lymphoid Tissues. In: Pathology and Genetics of Tumors of Hematopoietic and Lymphoid Tissues . IARC Press: Lyon; 2001.

5. Richter MN. Generalized Reticular Cell Sarcoma of Lymph Nodes Associated with Lymphatic Leukemia. Am J Pathol . 1928;4(4):285-292.

6. Tsimberidou A-M, Keating MJ. Richter syndrome: biology, incidence, and therapeutic strategies. Cancer . 2005;103(2):216-228.

7. Bockorny B, Codreanu I, Dasanu CA. Hodgkin lymphoma as Richter transformation in chronic lymphocytic leukaemia: a retrospective analysis of world literature. Br J Haematol . 2012;156(1):50-66.

8. Rossi D, Gaidano G. Richter syndrome: pathogenesis and management.Semin Oncol . 2016;43(2):311-319.

9. Tsimberidou A-M, O'Brien S, Khouri I, et al. Clinical outcomes and prognostic factors in patients with Richter's syndrome treated with chemotherapy or chemoimmunotherapy with or without stem-cell transplantation. J Clin Oncol Off J Am Soc Clin Oncol . 2006;24(15):2343-2351.

10. Jain P, Keating M, O'Brien S. Richter's syndrome - update on biology and management. Expert Opin Orphan Drugs . 2014;2(5):453-463.

11. Yamamoto K, Yakushijin K, Sanada Y, Kawamoto S, Matsuoka H, Minami H. Coexistent t(8;21)(q22;q22) Translocation and 5q Deletion in Acute Myeloid Leukemia. J Clin Exp Hematop JCEH . 2015;55(3):181-185.

12. Al-Harbi S, Aljurf M, Mohty M, Almohareb F, Ahmed SOA. An update on the molecular pathogenesis and potential therapeutic targeting of AML with t(8;21)(q22;q22.1);RUNX1-RUNX1T1. Blood Adv . 2020;4(1):229-238.

13. Lam K, Zhang D-E. RUNX1 and RUNX1-ETO: roles in hematopoiesis and leukemogenesis. Front Biosci J Virtual Libr . 2012;17:1120-1139.

14. Sood R, Kamikubo Y, Liu P. Role of RU NX 1 in hematologic malignancies. Blood 2017:129(15): 20702082

15. Libermann TA, Pan Z, Akbarali Y, Hetherington CJ, Boltax J, Yergeau DA, Zhang D. AML1 (CBF $\alpha 2$ ) Cooperates with B Cell-specific Activating Protein (BSAP/PAX5) in Activation of the B Cell-specific BLK Gene Promoter. J Biol Chem 1999; 274(35): 24671-24676

16. Grossmann V, Kern W, Harbich S, Alpermann T, Jeromin S, Schnittger S, Haferlach T, Kohlmann A. Prognostic relevance of RUNX 1 mutations in T-cell acute lymphoblastic leukemia. Haematologica 2011; 96(12): 1874-1877.

Figure 1: Peripheral blood showing prolymphocytic transformation to CLL cells (1A) and bone marrow biopsy with transformation of CLL to prolymphocytic leukemia (1B). 
Figure 2: Cytogenetics showed an abnormal male karyotype with six out of twenty metaphases showing a translocation between chromosomes 8 and 21 in addition to trisomy $12(2 \mathrm{~A})$, while remaining metaphases showed a normal karyotype (2B)
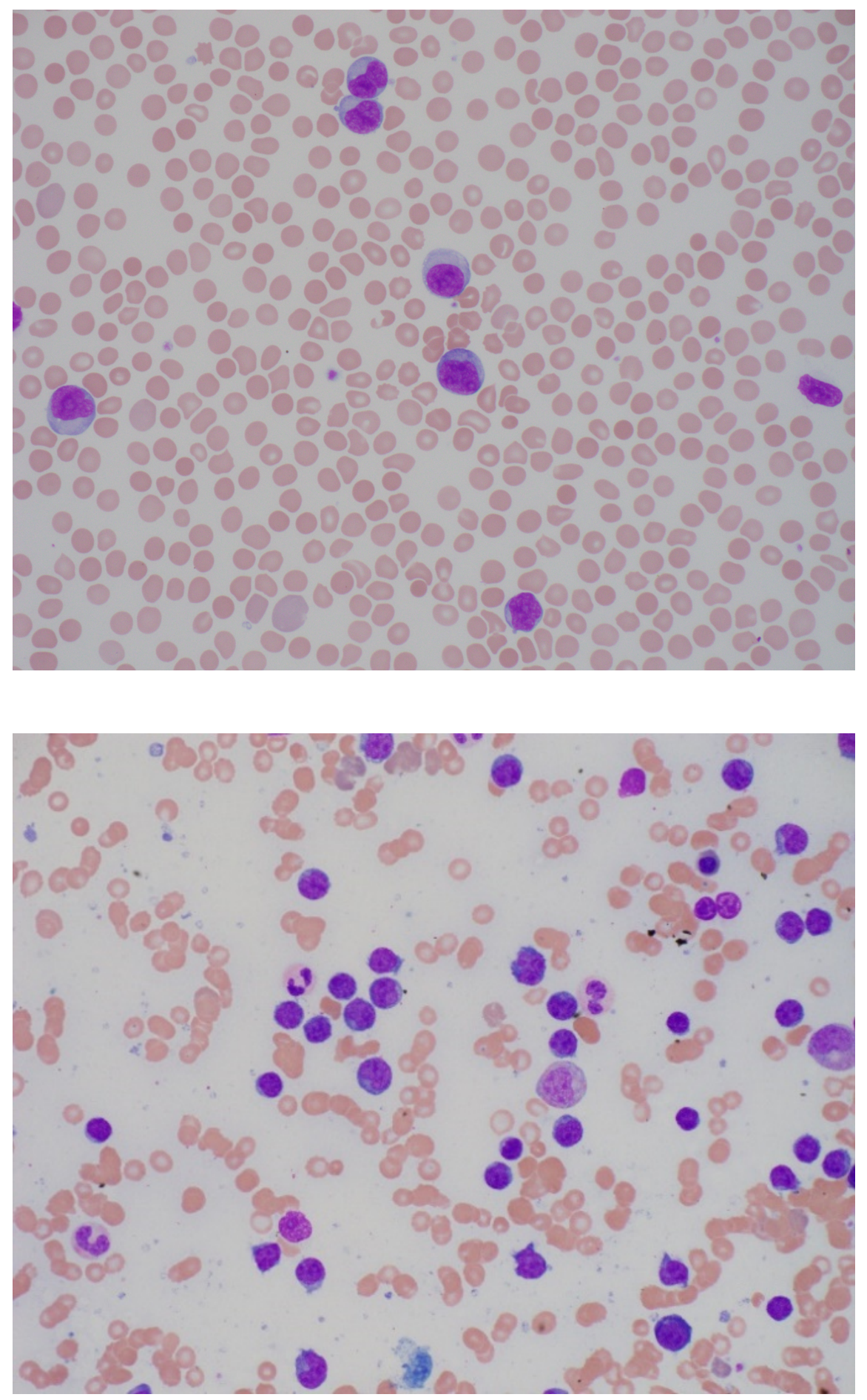

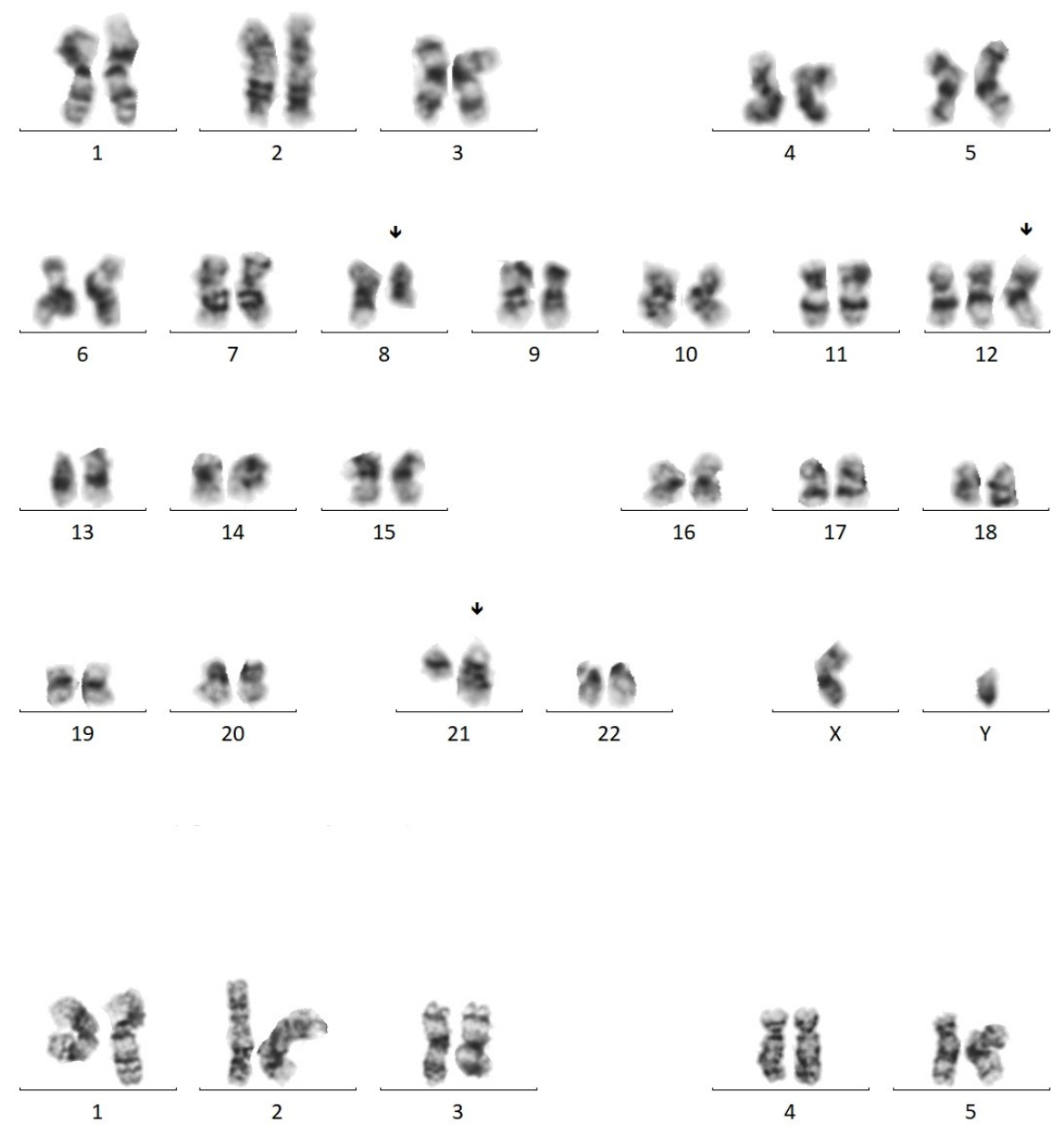

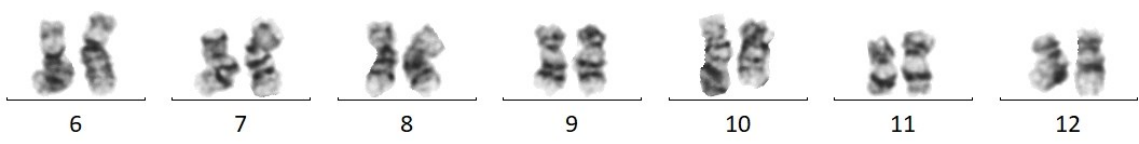

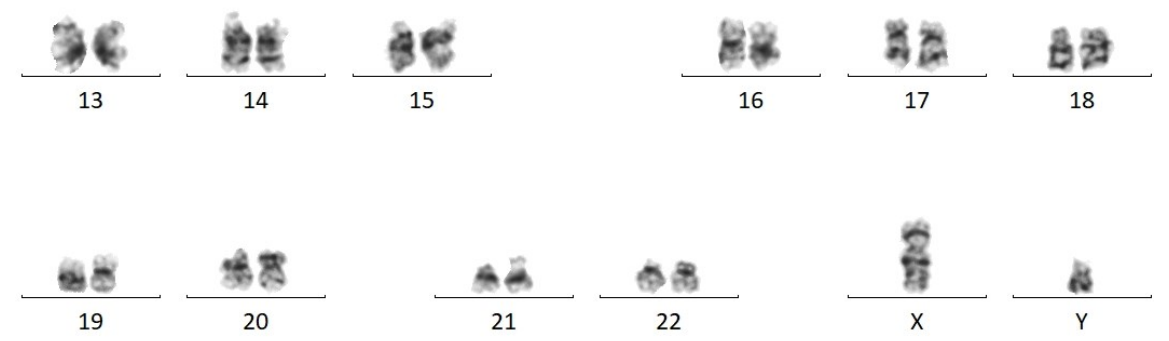

